\title{
Evolución e impacto en la comunicación de valores responsables. Caso de estudio en España
}

\author{
Belén LóPEZ VÁzQUEZ \\ ESIC, Business \& Marketing School \\ belen.lopez@esic.edu
}

\begin{abstract}
Resumen:
Se propone en este artículo un análisis de la evolución de la estrategia de Responsabilidad Social Corporativa (RSC) y Sostenibilidad de las corporaciones y su impacto en la sociedad. Esta estrategia corporativa se caracteriza por los impactos económicos, sociales y medioambientales que genera la actividad empresarial, así como los beneficios que reporta a los diferentes stakeholders. Las compañías, a su vez, utilizan diferentes herramientas de comunicación para informar a los grupos de interés sobre su gestión responsable. Se estudia en este artículo una empresa líder en España, Iberdrola, y su estrategia global de RSC. Para ello, se analizan las herramientas de comunicación estratégica que utiliza esta empresa para generar un diálogo con los grupos de interés. Todo ello se explora a través de los contenidos que ofrece la empresa en la web corporativa, que contiene información relevante, como: la memoria de sostenibilidad; los perfiles de la compañía en medios sociales como Facebook, Linkedn, Youtube; blogs corporativos, etc., que incluyen información corporativa, así como otra información corporativa relevante y accesible en la web a los stakeholders.

El objetivo de este análisis es conocer la relación entre las demandas sociales y las acciones socialmente responsables de la empresa, cuya finalidad es mostrar cómo contribuye la empresa al desarrollo sostenible. Como resultado de esta estrategia y la comunicación de los resultados de la compañía a los stakeholders, esta compañía incrementa la reputación en el mercado nacional e internacional. Esta empresa, al igual que otras empresas del IBEX-35, utiliza diferentes formatos para comunicar los impactos empresariales, si bien puede incrementar la participación de los grupos de interés considerando las múltiples oportunidades que ofrecen los medios sociales. Por último, este caso de estudio contribuye a enriquecer el debate sobre la responsabilidad social y su impacto en el desarrollo sostenible. El reto fundamental de las empresas es fomentar la participación de los stakeholders en la evolución y beneficio de la sociedad.
\end{abstract}

Palabras clave: Responsabilidad Social Corporativa; Sostenibilidad; Comunicación; Reputación corporativa.

\section{The evolution and impact of responsible communication values. A case study in Spain}

\begin{abstract}
:
This paper is an analysis of the evolution of strategy Corporate Social Responsibility (CSR) and Sustainability of corporations and their impact on society. This corporate strategy is characterized by economic, social and environmental impacts generated by the corporations, as well as the benefits to the various stakeholders. The companies, in turn, use different communication tools to inform to the stakeholders about their responsible strategy. This article analyze a leader company, Iberdrola, and its overall CSR strategy, through the analysis of strategic communication tools that this company uses to
\end{abstract}


generate a dialogue with the stakeholders. And this strategy is explored through the content that this company offer on the corporate website, which contains relevant information, such as: the sustainability report, the company profiles on social media like Facebook, Linkedn, YouTube, corporate blogs, etc., that include corporate information, and other relevant corporate information accessible on the corporate website to the stakeholders.

The objective of this analysis is to understand the relationship between social demands and the initiatives of socially responsible companies, and then show how the company contributes to the sustainable development. As a result of this strategy and the communication to the stakeholders, this company increase reputation in domestic and international markets. This corporation, like other companies in the IBEX -35, uses different channels to communicate business impacts, although it may increase the participation of stakeholders considering the diferent opportunities of social media. Finally, this case study contributes to enrich the debate on corporate social responsibility and its impact on sustainable development. The principal challenge for the companies is to promote the participation of stakeholders in the growth and benefit of society.

Key Words: Corporate Social Responsibility; Sustainability; Communication; Corporate Reputation.

\section{Referencia normalizada:}

López Vázquez, B. (2014): Evolución e impacto en la comunicación de valores responsables. Caso de estudio en España. Historia y Comunicación Social. Vol. 19. Núm. Especial Marzo. Págs. 511-523.

Sumario: 1. Introducción, 2. Revisión de la literatura, 3. Caso de estudio: Sostenibilidad en Iberdrola, 4. Una empresa responsable, 5. Conclusiones, 6. Bibliografía

\section{Introducción}

¿Es la RSC una estrategia de negocio que genera beneficios a los stakeholders? ¿Favorecen las empresas un cambio en la sociedad con las acciones socialmente responsables? ¿Qué actividades sostenibles comunican las compañías? ¿Generan las corporaciones la participación de los stakeholders en la empresa? Estas preguntas forman parte del debate de la RSC y la sostenibilidad y las respuestas tienen el objetivo de mostrar que existe una relación entre las demandas de los stakeholders a las empresas, el compromiso de las compañías con cada uno de sus grupos de interés y el impacto que las empresas generan en la sociedad con sus iniciativas económicas, sociales y medioambientales (Kujala, Penttilä, Tuominen, 2011).

Mediante el análisis de un caso de estudio, la multinacional española Iberdrola, compañía energética líder mundial en energía eólica, presente en casi 40 países, se analiza la información disponible en la web corporativa en el área de la sostenibilidad y la RSC. Para ello, se estudia la estrategia de sostenibilidad de esta corporación, las herramientas que la empresa utiliza para comunicar las actividades sostenibles, así como el contenido de esa información en la web corporativa. De este modo, se muestra la relación entre valores sostenibles de la empresa y formatos de comunicación 
que la empresa utiliza para informar a los stakeholders (Capriotti, Moreno, 2007). Respondiendo a estas cuestiones sumamos una aportación académica sobre la contribución de la empresa en el desarrollo sostenible, la comunicación de las acciones responsables de la compañía y la estrategia de la empresa para lograr la participación de los públicos en el ámbito de la sostenibilidad Schleggelmich, Pollach, 2005; Morsing, Schultz, 2006; Villagra, López, 2013).

Este articulo hace varias contribuciones. En primer lugar, se hace una aportación a la literatura académica en el ámbito de la RSC y su relación con la sostenibilidad, atendiendo a las demandas actuales de la sociedad y su desarrollo futuro. En segundo lugar, los retos en la relación entre la estrategia sostenible y la estrategia de comunicación de la compañía, que requiere el fomento de una mayor participación de los stakeholders. Y, en tercer lugar, consecuencia de los aspectos anteriores, el impacto que todo ello tiene en incrementar la reputación de la compañía (Garriga, Melé, 2004). De esta manera, RSC, sostenibilidad, comunicación corporativa y reputación corporativa sirven a la competitividad de las empresas en mercados globales, al tiempo que explican la estrategia que hoy adoptan las compañías en su compromiso con la sociedad.

Este artículo se estructura como sigue. En primer lugar, se hace una revisión de la literatura en materia de RSC, sostenibilidad, reputación y comunicación y la relación entre estos conceptos estratégicos en la empresa. A continuación, se presenta un análisis de Iberdrola en materia de sostenibilidad utilizando la metodología del caso de estudio, a través de un análisis de contenido de la web corporativa de la compañía. La siguiente sección se fundamenta en la discusión de la estrategia de esta compañía, desde la perspectiva de la empresa responsable. Y, por último, se presentan las conclusiones de este estudio considerando las nuevas demandas globales a las corporaciones y las contribuciones académicas en materia de sostenibilidad, RSC, reputación y comunicación corporativa.

\section{Revisión de la literatura}

El concepto de Responsabilidad Social Corporativa (RSC) se ha desarrollado a partir de la segunda mitad del SXX con la publicación de artículos que versaban sobre su definición, la importancia de entender la empresa como parte de la sociedad y la responsabilidad y ética de los directivos en su labor ejecutiva (Bowen, 1953). Desde esta perspectiva, se evalúa a la empresa por el conjunto de impactos generados por las diferentes actividades en su relación con los stakeholders. En este sentido, el tradicional papel de la empresa basado en principios económicos y cortoplacistas se reemplaza al incluir criterios de RSC basados en los efectos a largo plazo de la actividad empresarial (Carroll, 1991; Chiu, Sharfman, 2009; Christensen, Overdorf, 2000; Freeman, 1984, 1994, Aguinis, Glavas, 2012). Desde esta nueva perspectiva, la 
empresa socialmente responsable asume de forma voluntaria un conjunto de responsabilidades, como son: económicas, legales, éticas y filantrópicas, entendiendo la empresa como un ciudadano corporativo que contribuye al desarrollo de la sociedad y a la competitividad empresarial (Carroll, 1991; Garriga, Melé, 2004; Kujala, Penttilä, Tuominen, 2011). En la segunda década del siglo XX y con el avance del desarrollo científico, la RSC se relaciona con el desarrollo sostenible (WCED, 1987; Rudnicki, 2000). Temas como el cambio climático, los derechos humanos o la educación universal se convierten en asuntos globales que afectan a las empresas. De esta forma, las empresas incluyen estrategias para reducir los impactos negativos generados por la actividad empresarial y asumen la responsabilidad de modificar la estrategia empresarial en su actividad para su contribución positiva de los problemas que afectan al planeta (Elkington, 1999). De este modo, la sostenibilidad es un nuevo paradigma donde empresas, instituciones y sociedad de manera conjunta, trabajan alineadas para garantizar un futuro mejor mediante iniciativas globales que promueven instituciones como Naciones Unidas (Global Compact). Así, la sostenibilidad corporativa es la respuesta estratégica de las empresas ante las nuevas demandas globales (Edgeman, Hensler, 2001; Bansal, 2005, Bhattacharyya 2010, Asif, Searcy, Zutshi, Amad, 2011).

De manera concreta, en el entorno europeo se define RSC considerando un marco europeo competitivo común para las empresas que de manera voluntaria integran la RSC en su gestión (CEC, 2001). A su vez, la Comisión Europea señala 10 años después en su nueva definición (EC, 2011) que RSC es la responsabilidad de la empresa por los impactos que genera su actividad y la consecuencia que esto aporta en cada uno de los stakeholders generando un valor compartido de los beneficios empresariales. De este modo, se produce una evolución en el concepto de RSC considerando los múltiples impactos de la actividad empresarial, así como el valor compartido con todos los stakeholders (Porter, Kramer, 2011), manteniéndose el carácter voluntario de la RSC y ligado a la sostenibilidad en la estrategia empresarial Schleggelmich, Pollach, 2005; Morsing, Schultz, 2006; Villagra, López, 2013).

De esta manera, la vinculación de la sostenibilidad con la empresa es directa y, en segundo lugar, con los stakeholders, y en el compromiso que la empresa asume con cada uno de ellos. En la medida en la que la empresa cubre las expectativas de sus grupos de interés, se incrementa a su vez la reputación (Fombrum, 2005) y la confianza en la empresa. Desde esta perspectiva, los compromisos de las compañías traducidos en acciones concretas tienen una doble función: de manera externa, servir a la propia sociedad y, como consecuencia de ello, aportar beneficio interno a la empresa incrementando la reputación de la empresa. La reputación trae consigo consecuencias positivas para las compañías (Gray, Balmer, 1998: Chun, 2005; Fombrum, 2005), por lo que, es objetivo de las empresas formar parte de índices nacionales e internacionales de reputación corporativa (MERCO en España y Reputation Institute en USA, entre otros). Estos índices incluyen la RSC y la gestión ética de las compañías, como variables de reputación medibles (Chun, 2005; Fombrum, 2005).

Por su parte, la estrategia de comunicación que utilizan las empresas para comunicar sus resultados anuales en las diferentes áreas de las empresas se desarrolla mediante 
diferentes herramientas de comunicación. El principal documento es el informe de sostenibilidad, donde la empresa recoge los datos anuales económicos, sociales y medioambientales siguiendo indicadores de diversos estándares, como es Global Reporting Initiative, para dar información de los impactos de la empresa (Isaksson, R., Steimle, U., 2009). A su vez, las compañías utilizan otras herramientas para comunicar las acciones de RSC, como es la web corporativa, entre otras (Capriotti, Moreno, 2007). Ahora bien, se ha cuestionado la efectividad sobre la comunicación de valores y acciones de RSC, ya que, en ocasiones, esta comunicación despierta recelo y es percibida de modo negativo por los stakeholders (Villagra, López, 2013). Por lo que, si bien la comunicación es relevante para dar a conocer qué acciones realizan las compañías, no menos importante es la estrategia de medios que siguen las empresas en su comunicación de valores (Schleggelmich, Pollach, 2005). La finalidad es informar a los públicos para dar a conocer cómo se compromete la empresa en el desarrollo de la sociedad y, como consecuencia de estas acciones, obtener credibilidad como consecuencia de los hechos relevantes de la empresa (Keller, 2000; Schleggelmich, Pollach, 2005; Morsing, Schultz, 2006; Jahdi, Acikdili, 2009). De este modo, una estrategia de comunicación adecuada sirve también para diferenciar a la empresa de sus competidores en la comunicación de la estrategia sostenible de la compañía. En dicha estrategia, las compañías incluyen: tipo de mensaje, formato de comunicación, qué decir y cómo decirlo para obtener un efecto positivo y reforzar en sus públicos el compromiso sostenible de la empresa. Sin embargo, la comunicación contribuye a la diferenciación y competitividad de la empresa siempre y cuando la compañía defina con claridad su posicionamiento estratégico ligado a la sostenibilidad y utilice la comunicación para informar y motivar un cambio en la sociedad. De de esta forma, la empresa es percibida no sólo por el impacto y crecimiento económico propio de su actividad, si no también como ciudadano corporativo que genera beneficios múltiples en el conjunto de la sociedad. Las nuevas demandas de la sociedad apuntan a que la empresa también asume un papel activo en la resolución de los problemas globales como los derechos humanos, la educación, la salud y otros (Scherer, Palazzo, 2011). Una empresa comprometida con la sociedad es aquélla que, además de realizar actividades para incrementar los impactos positivos en el entorno, contribuye al cambio de la sociedad de manera activa con la participación de los stakeholders en iniciativas y propuestas conjuntas (Scherer, Palazzo, 2011). Los medios sociales ofrecen a las empresas la posibilidad de establecer un diálogo participativo con el objetivo de dar soluciones a problemas globales. Estas plataformas de comunicación ofrecen esta oportunidad de colaboración cuya consecuencia es el incremento de la credibilidad y la confianza al reforzar la relación con los grupos de interés. 


\section{Caso de estudio: Sostenibilidad en Iberdrola}

Para el desarrollo de este artículo se utiliza la metodología del caso de estudio (Ying, 1994) y, en particular, de una compañía multinacional española con más de 150 años de actividad dedicada a la sostenibilidad y el medio ambiente, a través de un análisis de contenido de la información que aparece en la web corporativa. Iberdrola es el primer grupo energético español, una de las principales empresas españolas del Ibex 35 por capitalización bursátil y empresa líder mundial del sector eólico. A través de su web corporative (figura 1), los stakeholders pueden encontrar amplia información sobre la historia, visión y misión de la empresa, un apartado de reputación y sostenibilidad, con el posicionamiento de la empresa en energías renovables, así como la estrategia global de la compañía. La sala de prensa destaca noticias en diferentes medios nacionales e internacionales donde aparece la compañía. Tienen, además, un apartado con información para accionistas e inversores, destacando su política de gobierno corporativo e informes anuales con datos económicos de la empresa. Cuenta además con un apartado para clientes, con tarifas y recomendaciones para el ahorro de energía y, por último, información sobre el modelo de gestión con proveedores y redes, que incluye una oficina virtual y redes inteligentes, entre otras informaciones.

De manera específica, se analiza el contenido de la contribución de la empresa en el área de sostenibilidad y reputación de la web corporativa, que incluye:

Nuestros compromisos, donde se definen visión y valores y los compromisos con cada uno de los stakeholders de la compañía. Además, se adjunta la política de Responsabilidad Social Corporativa de la compañía.

Principales iniciativas e índices. En materia de sostenibilidad y reputación, la empresa está incluida en los siguientes índices: Dow Jones Sustainability Indices, CDP Climate Disclosure Leader 2013, FTSE4Good, Global Roundtable on Climate Change, ROBECO SAM Sustainability Award Gold Class 2013, Carbon Ranking Global 800, NYSE Euronext, Award for Leadership in new energy 2013, European Business Awards 2012, 2013, MERCO, Monitor Empresarial de Reputación Corporativa, Oekom Research, The new economy, 2012, International Green Awards, 2012, entre otros. 
Fig. 1 Web corporativa de Iberdrola. Consulta realizada el 12 diciembre 2013.

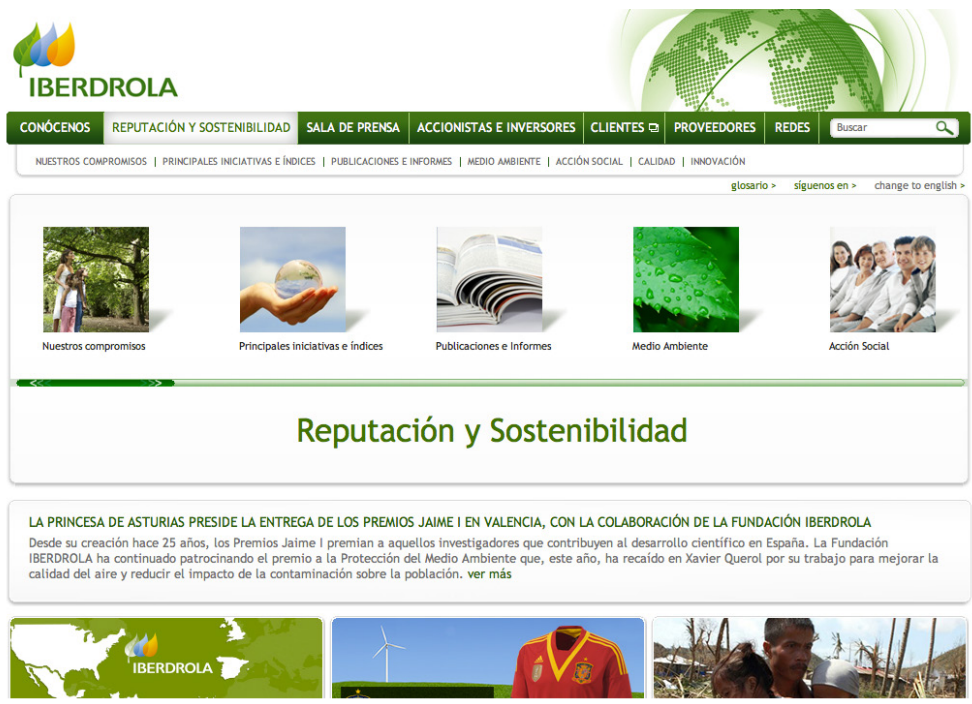

Entre los principales reconocimientos de la compañía en materia de sostenibilidad (figura 2), podemos destacar: empresa líder mundial del DJSI 2012 . Primera utility con activos nucleares seleccionada en FTSE4Good. Seleccionada también en CDLI y CDPI de Carbon Disclosure. Es una de las 100 compañías más sostenibles del mundo en Global 100 Gold Class del sector electricidad en Sustainability Yearbook 2012. Líder del sector utilities: electricidad, gas y agua, en Merco 2012.

Fig.2 Reconocimientos internacionales de Iberdrola.
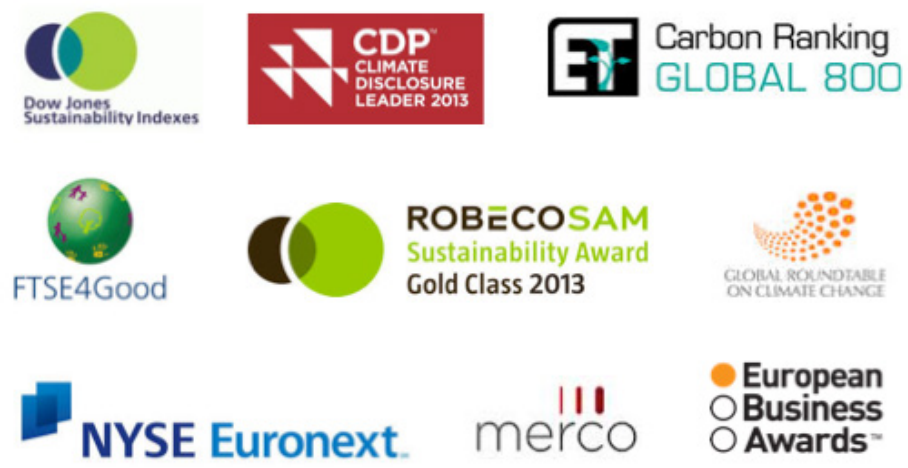

AWARD FOR LEADERSHIP N
IN NEW ENERGY

oekom relselarch

GREEN

AWARDS

\section{NEWECONOMY}


En el apartado de Principales publicaciones e Informes, podemos encontrar los informes de sostenibilidad de la compañía desde 2003 a 2012, disponibles en la web y con detallada información sobre indicadores económicos, sociales y medioambientales de su actividad cada año. Cuenta además con la certificación A+ de GRI por su completa información en la memoria de 2012. Además, la compañía proporciona los informes de progreso del Pacto Mundial y la revista Energía Sostenible, que incluye reportajes sobre la estrategia y logros de la compañía.

En la siguiente sección, Medioambiente, la organización alude al respeto al medio ambiente que recoge en el apartado de la vision de la compañía y donde se detalla la estrategia medioambiental de la empresa. En el punto siguiente, acción social, destaca el programa de voluntariado corporativo, entre otros, y la Fundación Iberdrola, centrada en proyectos en los ámbitos de la formación y la investigación, la sostenibilidad energética y la biodiversidad, el arte y la cultura, así como la solidaridad y la acción social. Otra pestaña es Calidad y, por último, Innovación destinada a la eficiencia, estrategia tecnológica y sostenibilidad. En este apartado, la compañia publica que ha destinado 145 millones de euros en 2012, un 6.6\% más que el año anterior.

Además, la Web corporativa cuenta con un apartado sobre cambio climático. En este apartado se ofrece información sobre qué es la huella de carbón e iniciativas ecoeficientes, destacando que la empresa evita 52 toneladas de emisiones de $\mathrm{CO} 2$ gracias a la movilidad sostenible. Además de la web corporativa, la empresa cuenta también con una activa presencia en medios sociales. De hecho, tienen perfil en Twitter, YouTube, Slideshare, Google+, Facebook, Flickr, Linkedln, Unience y cuenta también con 1 blog corporativo.

Por otra parte, Iberdrola comunica sus valores, compromisos y retos de futuro sostenibles mediante campañas de publicidad, que se pueden descargar de la web corporativa, donde muestran el compromiso con accionistas, la aportación a la sociedad y la apuesta por la innovación y las tecnologías renovables.

\section{Una empresa responsable.}

Tras el desglose de la información que aporta la empresa en la web corporativa, debe considerarse que la compañia comunica los impactos económicos, sociales y medioambientales de su actividad, año a año, tanto en los países que opera, como de los compromisos y actividades con stakeholders, recogidos en la memoria de sostenibilidad. Si bien es fácil localizar la información, debido al volumen del informe, sin embargo esta información debería ser más accessible a todos los stakeholders, en el sentido de que es una información especializada y extensa que podría ofrecerse de manera abreviada destacando los datos más relevantes de la compañía, ya que la accesibilidad implica también sencillez y claridad en la información. En ese sentido, la 
informacion puede suministrarse presentando la evolución de los impactos mediante un detalle sencillo sobre los resultados más destacables. Si bien la compañía cuenta con infinidad de reconocimientos, la comunicación se puede mejorar mediante la simplificación de la información técnica que requieren analistas e inversores, para informar a clientes y sociedad en general mediante la información más relevante. La utilización de gráficos para explicar la estrategia, es una forma rápida de obtener la información actualizada de la compañia.

Desde esta perspectiva, si bien Iberdrola es una empresa responsable con la sociedad y el entorno, se hace necesario conocer de forma comprensible a todos los stakeholders cómo está beneficiando la compañía a la sociedad. Caben destacar las acciones de la empresa para fomentar una sociedad más sostenible, como son: la movilidad verde, ideas para ahorrar energía, etc. Por lo que, si bien su contribución sostenible es positiva, cabe esperar en la empresa un mayor compromiso promoviendo una mayor concienciación en los ciudadanos, como ya lo están haciendo mediante actividades como es el voluntariado corporativo entre sus empleados, De este modo, el discurso empresarial se enlaza con el discurso social, entendiendo a la empresa como parte proactiva en la mejora de la sociedad. En este sentido, la empresa, entendida como empresa responsable (ver figura 3), puede liderar la participación de los stakeholders creando iniciativas y proyectos participativos con un fin social en medios sociales, que demuestren cómo benefician de manera concreta a la sociedad. Si bien la empresa comunica proyectos similares en el apartado de la Fundación Iberdrola, una empresa responsable es aquélla que promueve e incluye la participación de todos los stakeholders en la mejora de la sociedad.

Fig. 3 Modelo de empresa responsable. Elaboración propia.

MODELO EMPRESA RESPONSABLE
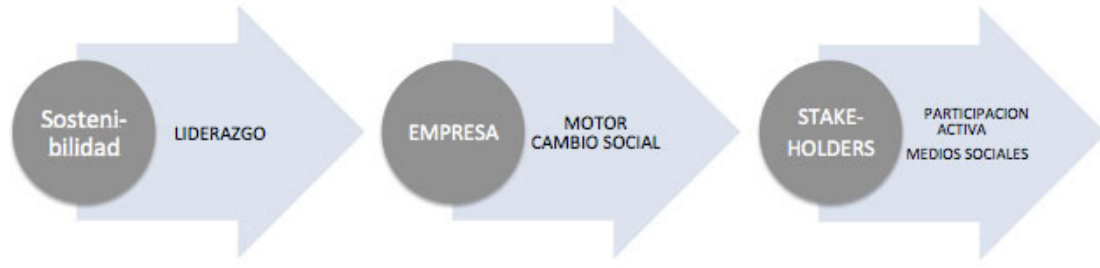

La verdadera contribución al desarrollo de la sociedad supone que la empresa asume un nuevo rol politico en la sociedad. Fomentar el uso del coche eléctrico, ideas para reducir el consumo de electricidad en el hogar, voluntariado corporativo entre los empleados, desarrollo de energías renovables, reducción de emisiones de $\mathrm{CO}$, etc., son ejemplos de acciones que tienen un impacto punctual pero que deben tener 
continuidad en el tiempo. La empresa responsable participa en el cambio global de la sociedad y se implica hoy en los problemas que afectan a la sociedad adoptando un papel activo y dando respuesta a temas globales, promoviendo la participación ciudadana y de este modo, siendo un referente para la mejora de la sociedad. Los medios sociales ofrecen una gran oportunidad a las compañías, tanto por su alcance como por su potencial de participación y colaboración con los stakeholders. Como consecuencia de promover el cambio social, la compañía incrementa credibilidad unida al compromiso medible de sus acciones.

\section{Conclusiones}

Este paper muestra cómo una empresa global desarrolla su estrategia corporativa ligada al ámbito de la RSC y su contribución al desarrollo sostenible. Para ello, utiliza diferentes herramientas de comunicación que están disponibles en la web corporativa, como son: La memoria de sostenibilidad, accessible desde 2003, donde se recoge información sobre indicadores económicos, sociales y medioambientales siguiendo los indicadores de GRI. Otra información corporativa, como es su presencia en indices de sostenibilidad y reputación. Iniciativas específicas medioambientales que la empresa lidera como son: las energías renovables y energía eólica, así como iniciativas tales como la movilidad sostenible y el fomento del ahorro energético.

El posicionamiento estratégico de la empresa está alineado con el contenido que ofrece la empresa, si bien, la empresa puede incrementar su nivel de participación mediante una utilización colaborativa con los stakeholders. Los medios sociales ofrecen esta posibilidad a la empresa para fomentar una comunicación bidireccional cuyo objetivo sea beneficiar a la sociedad en diferentes áreas. Si bien la empresa tiene perfil en diferentes plataformas on-line, proyectos colaborativos globales pueden ser desarrollados para lograr mayor impacto en la sociedad desde una perspectiva sostenible. De este modo, la empresa asume el papel participativo para el cambio social que los públicos demandan como parte del compromiso de las compañías. Una comunicación estratégica coherente y participativa tiene un efecto en la reputación, credibilidad y legitimidad de la compañía.

Si bien las limitaciones de un caso de estudio son evidentes para obtener conclusiones más generales sobre el impacto de las corporaciones en su estrategia sostenible, este artículo sin embargo hace una contribución académica en la comprensión de cómo estrategia sostenible, responsabilidad social corporativa, comunicación corporativa y reputación corporativa requieren una estrategia conjunta en la empresa responsable. Investigaciones futuras deben dar respuesta a cuáles son las formas más adecuadas de participación y colaboración de las empresas con los stakeholders para beneficiar a la sociedad a largo plazo. 


\section{Bibliografía}

AGUINIS, H., \& GLAVAS, A. (2012). "What We Know and Don't Know About Corporate Social Responsibility: A Review and Research Agenda". Journal of Management, 38(4), pp. 932-968.

ASIF, M., SEARCY, C., ZUTSHI, A., AHMAD, N. (2011). "An integrated management systems approach to corporate sustainability". European Business Review, Vol. 23 No. 4, pp. 353-367.

BANSA, P. (2005), "Evolving sustainably: a longitudinal study of corporate sustainable development", Strategic Management Journal, Vol. 26 No. 3, pp. 197-218.

BHATTACHARYYA, S. (2010), "Exploring the concept of strategic corporate social responsibility for an integrated perspective", European Business Review, Vol. 22 No. 1, pp. 82-101.

BOWEN,H.R.(1953). "Social responsibilities of the businessman". NewYork Harper \&Row.

CAPRIOTTI, P., MORENO, A. (2007) "Communicating corporate responsibility through corporate web sites in Spain", Corporate Communications: An International Journal, Vol. 12 Iss: 3, pp.221 - 237

CARROLL, A. B. (1979). "A Three-Dimensional Conceptual Model of Corporate Performance". Academy of Management Review, 4(4), 497-505.

CARROLL, A. B. (1991). "The Pyramid of Corporate Social Responsibility: Towards the Moral Management of Organizational Stakeholders". Business Horizons, July/ August, 39-48.

CHIU, S.-C., \& SHARFMAN, M. (2009). "Legitimacy, Visibility, and the Antecedents of Corporate Social Performance: An Investigation of the Instrumental Perspective". Journal of Management.

CHRISTENSEN, C., \& OVERDORF, M. (2000). "Meeting the challenge of disruptive change". Harvard Business Review, 78(2), 66-75.

CHUN, R. (2005): "Corporate reputation: Meaning and measurement". International Journal of Management Reviews 7 (2), 91-109

COMMISSION OF THE EUROPEAN COMMUNITIES (2001): "Green paper. Promoting a European framework for Corporate Social Responsibility". http:// eurlex.europa.eu/LexUriServ/site/en/com/2001/com2001_0366en01.pdf

EDGEMAN, R.L. HENSLER, D.A. (2001), "The AO chronicle: earth@omega or sustainability@alpha?”, The TQM Magazine, Vol. 13 No.2,pp. 83-90.

ELKINGTON, J. (1999), "Cannibals with Forks: The Triple Bottom Line of 21st Century" Business, Capstone Publishing Ltd, Oxford.

EUROPEAN COMMISION (2011): “A renewed EU strategy 2011-14 for Corporate Social Responsibility". http://eur-lex.europa.eu/LexUriServ/LexUriServ. do?uri=COM:2011:0681:FIN:EN:PDF

FOMBRUM, C. J. (2005). "Building Corporate Reputation Through CSR initiatives: Evolving Standards". Corporate Reputation Review, 8(1), 7-11.

FREEMAN, R. E. (1984). "Strategic Management: A Stakeholder Approach". Pitman, Boston. 
FREEMAN, R. E. (1994). "The Politics of Stakeholder Theory: Some Future Directions". Business Ethics Quarterly, 4(4), 409-429.

GARRIGA, E., \& MELE, D. (2004). "Corporate Social Responsibility Theories: Mapping the Territory". Journal of Business Ethics, 53, 51-71.

GRAY, E.R., BALMER, J.M.T. (1998) Managing Corporate Image and Corporate Reputation, London: Long Range Planning.

JAHDI, K., ACIKDILLI, G., "Marketing Communications and Corporate Social Responsibility (CSR): Marriage of Convenience or Shotgun Wedding?", Journal of Business Ethics, 88, 2009, pp. 103-113.

KELLER, K. L., "Building and managing corporate brand equity". En SCHULTZ, Majken, HATCH, Mary Jo y LARSE, Morgens H. (Eds.), The Expressive Organization, Oxford University press: Oxford, 2000, pp. 116-137.

KUJALA, J., PENTTILÄ, K. y TUOMINEN, P., "Creating a conceptual model for building responsible brands", EJBO, Electronic Journal of Business Ethics and Organization Studies, 2011, Vol. 16, No.1, pp. 6-12.

MORSING, M. y SCHULTZ M., "Corporate social responsibility communication: stakeholder information, response and involvement strategies", Business Ethics: A European Review, vol. 15, nº 4, October 2006, pp. 323-339.

PORTER, M. E., \& KRAMER, M. R. (2011). Creating Shared Value. Harvard Business Review 89, nos. 1-2 (January-February 2011).

RUDNIKI, M., 'Environmental and Social Responsibility as the value in modern politics', Paper delivered during meeting the OIKOS Int. Warsaw, 10 November 2000.

SCHERER, A.G., PALAZZO G. (2011). "The New Political Role of Business in a Globalized World: A Review of a New Perspective on CSR and its Implications for the Firm, Governance, and Democracy." Journal of Management Studies, Volume 48, Issue 4, pages 899-931

SCHLEGELMILCH, B. B., POLLACH, I., "The perils and Opportunities of Communicating Corporate Ethics", Journal of Marketing Management, 21, 2005, pp. 267-290.

VILLAGRA, N., LOPEZ, B. (2013). "Analysis of values and communication of the Responsible Brands. Corporate Brand strategies for sustainability". Communication and Society/Comunicación y Sociedad, 26(1), 201-226.

World Commission on Environment and Development (1987), Our Common Future, Oxford University Press, Oxford.

YIN, R. (1994). Case Study Research: Design and Methods. Beverly Hills: Sage. 


\section{La autora}

Doctora en Publicidad y Relaciones Públicas por la Universidad del País Vasco, es profesora de Comunicación y RSC en programas nacionales e internacionales y Coordinadora Internacional de Grado en ESIC, Business and Marketing School, Madrid.

Autora del libro "Publicidad emocional. Estrategias creativas", ESIC, 2007, ha publicado artículos en revistas académicas y profesionales. Participa en congresos académicos nacionales e internacionales, siendo sus principales líneas de investigación: la gestión de intangibles, la comunicación corporativa y la RSC, como modelo de gestión empresarial.

Belén López ha sido investigadora visitante en la Universidad de Bristol, UK. Además, ha sido profesora visitante en las universidades de Anhui, Universidad de Finanzas y Económicas, China, Plantijn Hogeschool University y Erasmus University College, ambas en Bélgica. También ha sido profesora de Comunicación en SISU, Shanghai International Studies University, Shanghai, China. Asociada a Academy of Management, Academy of International Business \& European Communication Research and Education Association. 